\title{
Chemical Design Principles for Cache-Type Sc-Sb-Te Phase-Change Memory Materials
}

\author{
Getasew M. Zewdie, ${ }^{\dagger}, \#$ Yuxing Zhou, ${ }^{\dagger, \# \odot ~ L i a n g ~ S u n, ~}{ }^{\ddagger}$ Feng Rao, ${ }^{\S}$ Volker L. Deringer, ${ }^{\| \odot}$ \\ Riccardo Mazzarello, ${ }^{\perp}$ and Wei Zhang*, ${ }^{*}+0$
}

$\dagger$ Center for Advancing Materials Performance from the Nanoscale, State Key Laboratory for Mechanical Behavior of Materials, Xi’an Jiaotong University, Xi'an 710049, China

${ }^{\ddagger}$ Key Laboratory of Materials Processing Engineering, College of Materials Science and Engineering, Xi'an Shiyou University, Xi'an 710065, China

${ }^{\S}$ College of Materials Science and Engineering, Shenzhen University, Shenzhen 518060, China

"Department of Engineering, University of Cambridge, Cambridge CB2 1PZ, U.K.

${ }^{\perp}$ Institute for Theoretical Solid-State Physics, JARA-FIT and JARA-HPC, RWTH Aachen University, Aachen 52056, Germany

Supporting Information

ABSTRACT: Enhanced crystal nucleation in a $\mathrm{Sc}-\mathrm{Sb}-\mathrm{Te}$ phase-change material has enabled subnanosecond switching in phase-change memory devices, making cachetype nonvolatile memory feasible. However, the microscopic mechanisms remain to be further explored. In this work, we present a systematic ab initio study of the relevant parent compounds, namely, $\mathrm{Sc}_{2} \mathrm{Te}_{3}$ and $\mathrm{Sb}_{2} \mathrm{Te}_{3}$. Despite similar bond lengths and angles in the amorphous phases of the two compounds, $\mathrm{Sc}_{2} \mathrm{Te}_{3}$ displays a much more ordered amorphous network without homopolar bonds. As a result, the local structural order in amorphous $\mathrm{Sc}_{2} \mathrm{Te}_{3}$ is dominated by square motifs, remarkably similar to those of the metastable rocksalt-like phase. Chemical bonding analysis indicates more robust $\mathrm{Sc}-\mathrm{Te}$ bonds compared with $\mathrm{Sb}-\mathrm{Te}$ bonds in the amorphous phase, as well as a substantial role of electrostatic interactions in $\mathrm{Sc}_{2} \mathrm{Te}_{3}$ but not in

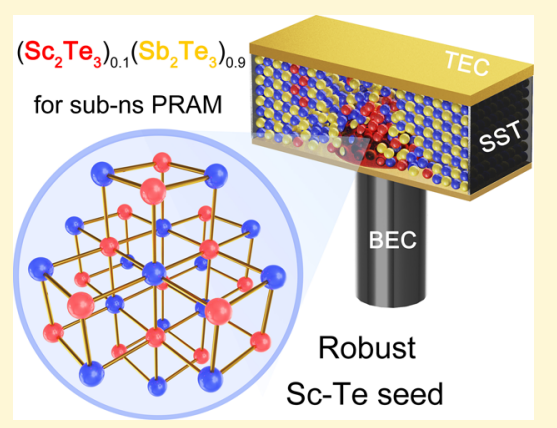
$\mathrm{Sb}_{2} \mathrm{Te}_{3}$. The robustness of $\mathrm{Sc}-\mathrm{Te}$ bonds explains the enhanced nucleation in $\mathrm{Sc}-\mathrm{Sb}-$ Te compounds. Finally, we discuss an alloying strategy of $\mathrm{Sc}_{2} \mathrm{Te}_{3}$ and $\mathrm{Sb}_{2} \mathrm{Te}_{3}$ for cache-type $\mathrm{Sc}-\mathrm{Sb}-\mathrm{Te}$-based phase-change memory.

\section{INTRODUCTION}

In the information era, the exponentially increasing demand for data storage and processing is posing a serious challenge for current silicon-based electronic devices. ${ }^{1} \mathrm{New}$ technologies and new materials are being pursued to cope with this problem. ${ }^{1}$ Nonvolatile memory ${ }^{2-6}$ and neuro-inspired computing $^{6-9}$ are two promising routes for substantial improvement of computing efficiency, as the former optimizes the memory hierarchy, and the latter unifies computing with storage in the same memory cell. Among the emerging materials for these two technologies, ${ }^{1}$ chalcogenide phase-change materials $(\mathrm{PCMs})^{2,6}$ stand out as the most mature candidate, owing to their robust working mechanism and their high compatibility with the complementary metal-oxide-semiconductor technology. ${ }^{10}$ Competitive phase-change memory products have been released recently, and are expected to lead to a major change in data-intensive applications. ${ }^{11}$

Phase-change memory exploits the large electrical resistance contrast between the amorphous and crystalline state of PCMs, such as $\mathrm{Ge}_{2} \mathrm{Sb}_{2} \mathrm{Te}_{5}$ (GST), ${ }^{12-16}$ to enable digital data storage. $^{2,3}$ The switching between the two states is accomplished by rapid and reversible structural transitions at elevated temperatures, leading to switching times on the order of tens of nanoseconds. ${ }^{17-20}$ Yet, the two states are thermally robust at room temperature for decades in the absence of power supply, ensuring long-term data storage. RESET operations (amorphization) can be accomplished within 1 nanosecond, whereas SET operations (crystallization) need much longer time. Preprogramming strategies were developed to speed up the SET process of GST devices to enter the subnanosecond regime. ${ }^{17,21}$ More recently, systematic screening across the periodic table led to the design of a new material, namely, $\mathrm{Sc}_{0.2} \mathrm{Sb}_{2} \mathrm{Te}_{3}$ ("SST" in the following), which was shown to enable subnanosecond SET operations even in the absence of a pretreatment. ${ }^{22}$ This intrinsic material approach extends the capability of phase-change memory to compete with cache memory-static random access memory in terms of both switching speed and operation frequency. Taking into account the nonvolatile feature of SST, this new material opens up the possibility to build a universal memory ${ }^{23}$ using phase-change devices.

Received: February 3, 2019

Revised: April 24, 2019

Published: April 25, 2019 

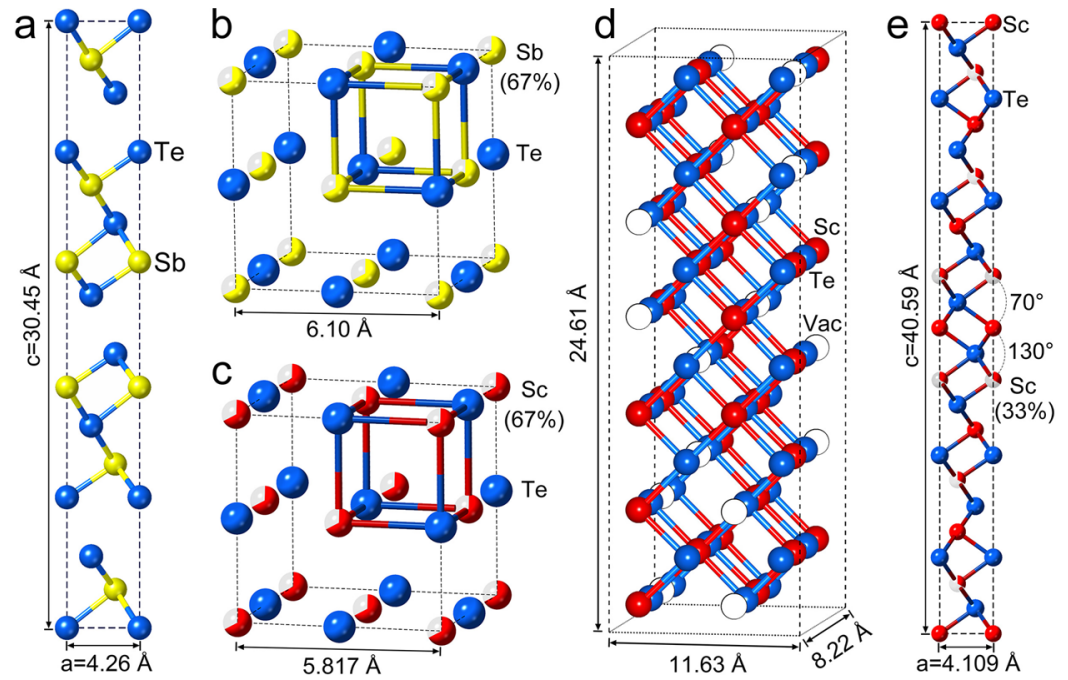

Figure 1. Crystal structures of $\mathrm{Sc}_{2} \mathrm{Te}_{3}$ and $\mathrm{Sb}_{2} \mathrm{Te}_{3}$. (a) Rhombohedral $\mathrm{Sb}_{2} \mathrm{Te}_{3}$ (in the conventional hexagonal cell setup). The $\mathrm{Sb}-\mathrm{Te}$ bond lengths are 2.97 and $3.13 \AA$. (b,c) Rocksalt-like $\mathrm{Sb}_{2} \mathrm{Te}_{3}$ and $\mathrm{Sc}_{2} \mathrm{Te}_{3}$. The $\mathrm{Sb}-\mathrm{Te}$ and $\mathrm{Sc}-\mathrm{Te}$ bond lengths are $\sim 3.05$ and $\sim 2.91 \AA$, respectively. 2/3 of the cation sites are occupied, as indicated by partially filled spheres. Four-membered rings as the defining structural motif are highlighted. (d) Orthorhombic $\mathrm{Sc}_{2} \mathrm{Te}_{3}$. Atomic vacancies are arranged in an ordered fashion. The $\mathrm{Sc}-\mathrm{Te}$ bond length is $\sim 2.90 \AA$. . (e) $\mathrm{Rhombohedral} \mathrm{Sc}_{2} \mathrm{Te}_{3}$. The occupancy for one of the $S c$ sites $(6 \mathrm{c})$ is $1 / 3$, as indicated by partially filled spheres, whereas the others ( $3 a$ and $3 b$ ) are fully occupied; see ref 37 . The Sc-Te bond length is $\sim 2.95 \AA$.

The technologically relevant properties of PCMs can be largely traced back to the atomic-scale structure and the dynamics of the microscopic constituents. The abundance of structural fragments that can serve as crystalline precursorsnamely, $\mathrm{ABAB}$ squares $(\mathrm{A}=\mathrm{Ge}$ or $\mathrm{Sb}, \mathrm{B}=\mathrm{Te})$ in amorphous GST - is one key parameter for fast incubation, ${ }^{24-29}$ whereas the dynamical stability of $\mathrm{ABAB}$ squares at elevated temperatures is another one. ${ }^{22}$ In supercooled liquid GST, $A B A B$ squares have a short lifetime. $B y$ contrast, $A B A B$ squares are much more robust in supercooled liquid SST, thus reducing the stochasticity of nucleation and leading to an ultrafast incubation process. ${ }^{22}$ However, there remain open questions regarding the chemical properties and crystallization dynamics in SST. First, different crystalline polymorphs are known for both $\mathrm{Sc}_{2} \mathrm{Te}_{3}$ and $\mathrm{Sb}_{2} \mathrm{Te}_{3}$; how do they affect the crystallization tendency of SST? Second, what are the structural properties of the amorphous phases, and what are the bonding mechanisms at play? Finally, why does the concentration of Sc in SST need to be kept at a relatively small percentage for high performance of phase-change devices?

In this work, we perform thorough structural and chemical bonding characterizations of both crystalline and amorphous (a-) $\mathrm{Sc}_{2} \mathrm{Te}_{3}$ and $\mathrm{Sb}_{2} \mathrm{Te}_{3}$, the two parent compounds of SST, based on ab initio simulations. We aim at understanding the nature of bonding in $\mathrm{a}-\mathrm{Sc}_{2} \mathrm{Te}_{3}$ and $\mathrm{a}-\mathrm{Sb}_{2} \mathrm{Te}_{3}$, and the way by which it leads to the enhanced nucleation in SST. These atomistic and chemical insights are expected to drive further development of cache-type phase-change memory.

\section{RESULTS AND DISCUSSION}

Crystalline Parent Compounds. Polymorphism occurs for both crystalline $\mathrm{Sb}_{2} \mathrm{Te}_{3}$ and $\mathrm{Sc}_{2} \mathrm{Te}_{3}$. The stable phase of $\mathrm{Sb}_{2} \mathrm{Te}_{3}$ is rhombohedral $(R \overline{3} \mathrm{~m})$ with alternately stacked $\mathrm{Sb}$ and Te layers forming quintuple blocks along the $c$ axis. ${ }^{30}$ Each unit cell contains three quintuple blocks and three gap layers (Figure 1a). In addition, a metastable defective rocksalt-like polymorph of $\mathrm{Sb}_{2} \mathrm{Te}_{3}$ was identified recently. ${ }^{31}$ This crystalline phase shares similar structural features as rocksalt-like
$\mathrm{GST}^{32-34}$ in that one sublattice is fully occupied by $\mathrm{Te}$ atoms, whereas the other one is randomly occupied by $2 / 3 \mathrm{Sb}$ atoms and $1 / 3$ vacancies (Figure $1 \mathrm{~b}$ ). Despite the high concentration of atomic vacancies, Peierls-like distortion around $\mathrm{Sb}$ atoms is found in this disordered rocksalt-like structure (Figure S1). In both polymorphs, $\mathrm{Sb}$ and $\mathrm{Te}$ atoms form heteropolar $\mathrm{Sb}-\mathrm{Te}$ bonds exclusively, with bond angles close to $90^{\circ}$. As regards $\mathrm{Sc}_{2} \mathrm{Te}_{3}$, three crystalline phases have been described. Similar to rocksalt-like $\mathrm{Sb}_{2} \mathrm{Te}_{3}$, a defective rocksalt-like phase of $\mathrm{Sc}_{2} \mathrm{Te}_{3}$ exists (Figure 1c), which has a smaller lattice parameter ${ }^{35}(a \approx 5.817 \AA)$ than the one of $\mathrm{Sb}_{2} \mathrm{Te}_{3}(a \approx 6.10 \AA) .{ }^{31}$ Another crystalline phase of $\mathrm{Sc}_{2} \mathrm{Te}_{3}$ forms an orthorhombic $\mathrm{Sc}_{2} \mathrm{~S}_{3}$-type structure with octahedral coordination. ${ }^{36}$ The only difference between this phase and the rocksalt-like phase is that the $1 / 3$ atomic vacancies on the cation sublattice are arranged in an ordered fashion (Figure 1d). The third crystalline phase of $\mathrm{Sc}_{2} \mathrm{Te}_{3}$ is a layered rhombohedral phase in space group $R \overline{3} m$ with alternating layers resembling cation-deficient B1-like ( $\mathrm{NaCl}$-type) and B8like (NiAs-type) motifs stacked along the $c$ axis; no gap layers are present here ${ }^{37}$ (Figure 1e). Some cation layers consist of $1 /$ $3 \mathrm{Sc}$ atoms and $2 / 3$ atomic vacancies. All Sc atoms are octahedrally coordinated by Te. By contrast, some Te atoms form $\mathrm{Sc}-\mathrm{Te}$ bonds with $70^{\circ}$ and $130^{\circ}$ bond angle near the B8like fragments, in addition to the majority of octahedral Sc-Te bonds.

Density functional theory (DFT) calculations are performed by using the Perdew-Burke-Ernzerhof (PBE) functional (see more details in the Methods section). They show that rocksaltlike $\mathrm{Sb}_{2} \mathrm{Te}_{3}$ is $\sim 75 \mathrm{meV} /$ atom higher in total energy than the stable rhombohedral phase. The calculated cohesive energies are -0.06 and $-0.12 \mathrm{eV} /$ atom for rocksalt-like and rhombohedral $\mathrm{Sb}_{2} \mathrm{Te}_{3}$, respectively. As regards $\mathrm{Sc}_{2} \mathrm{Te}_{3}$, all three crystalline phases have a substantially more favorable cohesive energy than $\mathrm{Sb}_{2} \mathrm{Te}_{3}$, that is $-1.23,-1.22$, and -1.15 $\mathrm{eV} /$ atom for orthorhombic, rhombohedral, and rocksalt-like $\mathrm{Sc}_{2} \mathrm{Te}_{3}$, respectively. More precisely, our calculations reveal that the rocksalt-like phase and rhombohedral phase that 


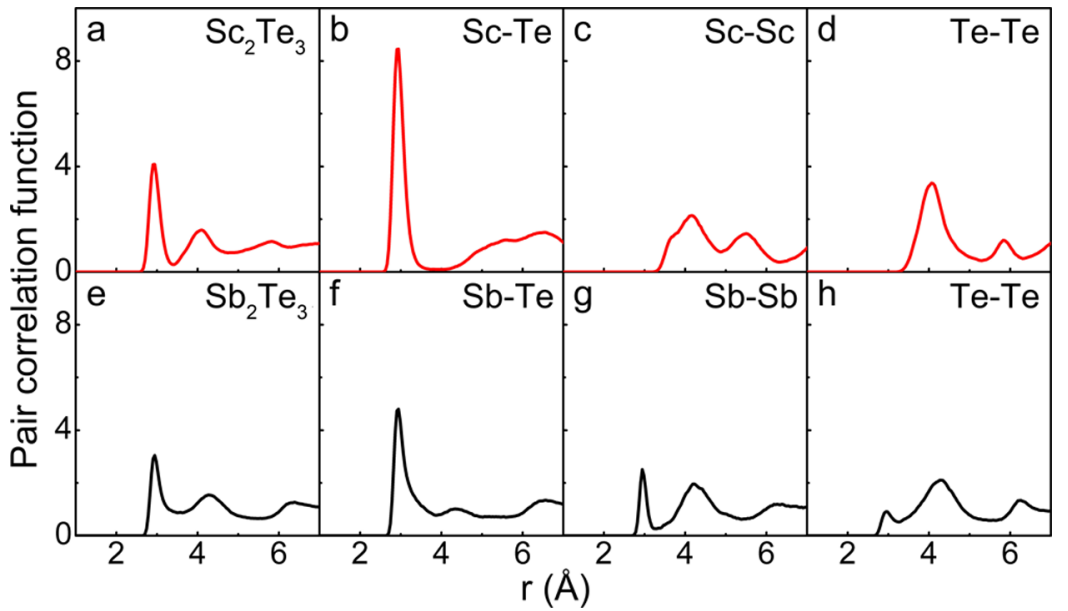

Figure 2. Atomic contacts in the amorphous phases, determined from the PCF. Data were collected based on a 10 ps AIMD trajectory at $300 \mathrm{~K}$. (a-d) Total and partial PCFs of a-Sc $\mathrm{Se}_{2}$. The positions of the first peak are $2.93 \AA$ (total), $2.93 \AA$ ( $\mathrm{Sc}-\mathrm{Te}$ ), $4.14 \AA$ ( $\mathrm{Sc}-\mathrm{Sc}$ ), and $4.06 \AA$ ( Te$\mathrm{Te})$, respectively. $(\mathrm{e}-\mathrm{h})$ Total and partial PCFs of $\mathrm{a}-\mathrm{Sb}_{2} \mathrm{Te}_{3}$. The position of the first peak is at $\sim 2.93 \AA$ for the total and the three partial PCFs.

contain strong disorder (i.e., randomly distributed atomic vacancies on the cation sublattice) are $\sim 84$ and $\sim 13 \mathrm{meV} /$ atom higher in total energy than the ordered orthorhombic phase (see Methods).

For $\mathrm{Sb}_{2} \mathrm{Te}_{3}$ and SST, a rocksalt-like structure is obtained upon rapid crystallization. ${ }^{22}$ However, the rhombohedral phase of $\mathrm{Sc}_{2} \mathrm{Te}_{3}$ deviates from the rocksalt-like phase with nonoctahedral structural features, which could be present in the amorphous state of $\mathrm{Sc}$-rich $\mathrm{Sc}-\mathrm{Sb}-\mathrm{Te}$ compounds as well. In terms of subnanosecond nucleation processes at evaluated temperatures, the nonoctahedral motifs might hinder the formation of nuclei, thus slowing down the SET operations of phase-change devices. Structural and bonding analyses of the amorphous phase discussed below provide further support that the concentration of $\mathrm{Sc}$ in $\mathrm{Sc}-\mathrm{Sb}-\mathrm{Te}$ compounds needs to be low.

Structure of the Amorphous Phases. An amorphous (a) $\mathrm{Sc}_{2} \mathrm{Te}_{3}$ structural model was generated in melt-quench simulations (Figure S2). A $3 \times 3 \times 3$ expansion of the rocksaltlike unit cell with the experimental lattice parameter was used as the starting configuration (180 atoms in a $17.58 \times 17.58 \times$ $17.58 \AA^{3}$ box). It was quickly heated above $2000 \mathrm{~K}$ for randomization, and then quenched down to the melting temperature of $\sim 1250 \mathrm{~K}$ and equilibrated there for $30 \mathrm{ps}$. Next, the liquid model was quenched down to $300 \mathrm{~K}$ within $50 \mathrm{ps}$ and equilibrated at $300 \mathrm{~K}$ for $30 \mathrm{ps}$ (Figure S2). The box size of the model was adjusted seven times during the quenching simulation to reduce the internal stress. A theoretical density of 0.0313 atom $\AA^{-3}$ was obtained for a- $\mathrm{Sc}_{2} \mathrm{Te}_{3}$, corresponding to a supercell of $17.91 \times 17.91 \times 17.91 \AA^{3}$. Two additional a$\mathrm{Sc}_{2} \mathrm{Te}_{3}$ models and three $\mathrm{a}-\mathrm{Sb}_{2} \mathrm{Te}_{3}$ models (containing 180 atoms each) were generated with the same scheme. The theoretical density of 0.0271 atom $\AA^{-3}$ was used for a$\mathrm{Sb}_{2} \mathrm{Te}_{3} .{ }^{38}$ The structural features of $\mathrm{a}-\mathrm{Sb}_{2} \mathrm{Te}_{3}$ discussed below are in good agreement with previous work. ${ }^{38-40}$ All three a$\mathrm{Sc}_{2} \mathrm{Te}_{3}$ models give very similar results, and the same holds for $\mathrm{a}-\mathrm{Sb}_{2} \mathrm{Te}_{3}$.

The (partial) pair correlation functions (PCFs) were calculated based on the ab initio molecular dynamics (AIMD) trajectories at $300 \mathrm{~K}$ (Figure 2). As regards the total PCF, a- $\mathrm{Sc}_{2} \mathrm{Te}_{3}$ (Figure 2a) shows a similar profile as compared with $\mathrm{a}_{-} \mathrm{Sb}_{2} \mathrm{Te}_{3}$ (Figure 2e), with similar peak positions for both the first $(\sim 2.9 \AA)$ and second peaks $(\sim 4.2$ $\AA$ ). This structural similarity guarantees the overall geometrical compatibility between the two amorphous materials. However, clear differences are observed at $\sim 3.4 \AA$, in that the first valley of the total PCF is deeper in a-Sc $\mathrm{Te}_{3}$ than in $\mathrm{a}-\mathrm{Sb}_{2} \mathrm{Te}_{3}$, indicating much fewer chemical bonds in this length range in the $\mathrm{Sc}$ compound. Indeed, there is almost no contact between Sc and Te atoms between 3.3 and $4.2 \AA$, whereas a finite fraction of $\mathrm{Sb}-\mathrm{Te}$ bonds is observed consistently in the same length range. Furthermore, a primary peak for homopolar $\mathrm{Sb}-$ $\mathrm{Sb}$ and $\mathrm{Te}-\mathrm{Te}$ bonds is found at $\sim 3.0 \AA$ in $\mathrm{a}-\mathrm{Sb}_{2} \mathrm{Te}_{3}$, but is absent in $\mathrm{a}-\mathrm{Sc}_{2} \mathrm{Te}_{3}$, indicating a more ordered amorphous network in $\mathrm{Sc}_{2} \mathrm{Te}_{3}$. Based on the PCF data, we set a uniform cutoff at $3.3 \AA$ for a- $\mathrm{Sc}_{2} \mathrm{Te}_{3}$, and separate cutoffs for $\mathrm{a}-\mathrm{Sb}_{2} \mathrm{Te}_{3}$ with $\mathrm{Sb}-\mathrm{Te} 3.4 \AA$, for $\mathrm{Sb}-\mathrm{Sb} 3.3 \AA$, and $\mathrm{Te}-\mathrm{Te} 3.2 \AA$, for further structural analyses. The choice of cutoffs for $\mathrm{a}-\mathrm{Sb}_{2} \mathrm{Te}_{3}$ is in line with previous work. ${ }^{38,39}$

Figure $3 \mathrm{a}$ shows the distribution of coordination numbers (CNs) in our systems. In a-Sc $\mathrm{Te}_{3}$, the majority of $\mathrm{Sc}$ and $\mathrm{Te}$
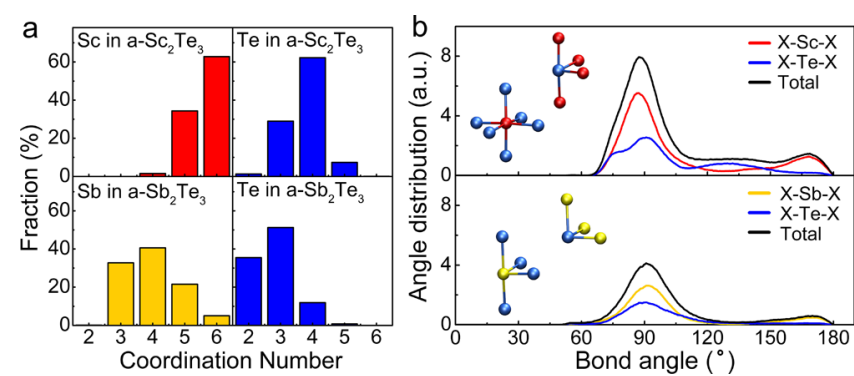

Figure 3. The local structural analysis for $a-\mathrm{Sc}_{2} \mathrm{Te}_{3}$ and $\mathrm{a}-\mathrm{Sb}_{2} \mathrm{Te}_{3}$ at 300 K. (a) Distribution of CN and (b) ADF of a-Sc $\mathrm{Te}_{3}$ and $\mathrm{a}-\mathrm{Sb}_{2} \mathrm{Te}_{3}$. Insets in (b) show typical atomic configurations for $\mathrm{Sc}(\mathrm{CN}=6)$ and $\mathrm{Te}(\mathrm{CN}=4)$ atoms in $\mathrm{a}-\mathrm{Sc}_{2} \mathrm{Te}_{3}$, and $\mathrm{Sb}(\mathrm{CN}=4)$ and $\mathrm{Te}(\mathrm{CN}=3)$ atoms in $\mathrm{a}-\mathrm{Sb}_{2} \mathrm{Te}_{3}$.

atoms are six- and four-fold coordinated, whereas in $\mathrm{a}-\mathrm{Sb}_{2} \mathrm{Te}_{3}$, most $\mathrm{Sb}$ and $\mathrm{Te}$ atoms are four- and three-fold coordinated. Note that, in the rocksalt-like phases, Sc and Sb have six-fold coordination, whereas $\mathrm{Te}$ atoms are, on average, four-fold coordinated due to the presence of vacancies. The angular distribution functions (ADF) show a prominent feature of octahedral configuration with a major peak around $90^{\circ}$ and a 


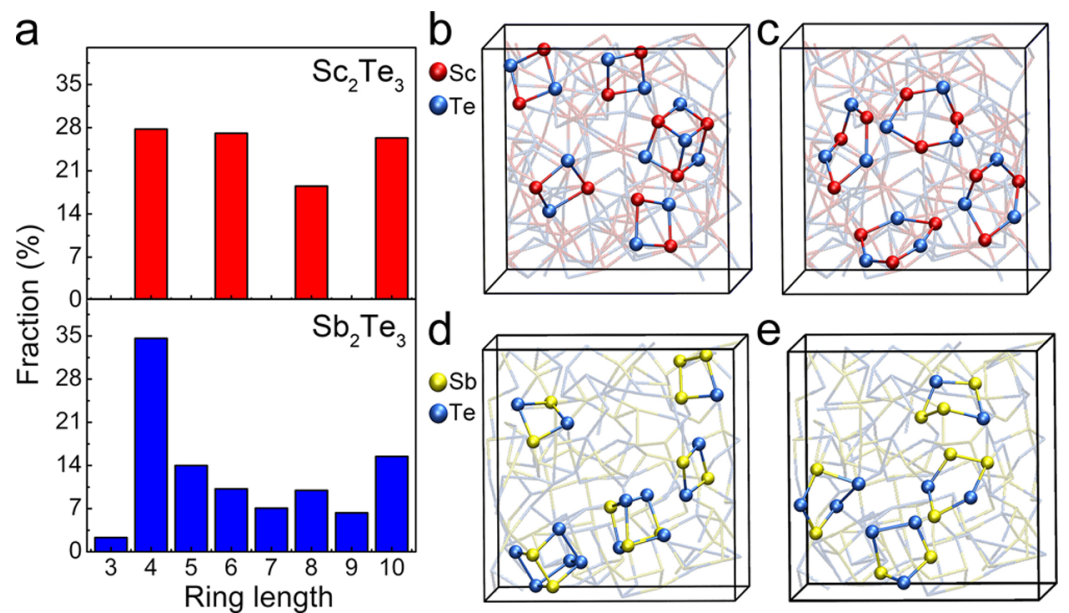

Figure 4. Medium range order in a-Sc $\mathrm{Te}_{3}$ and $\mathrm{a}-\mathrm{Sb}_{2} \mathrm{Te}_{3}$ at $300 \mathrm{~K}$. (a) Statistics for primitive rings: note the absence of any odd-membered rings in the Sc phase. (b-e) Snapshots of typical four- and six-membered primitive rings in a-Sc $\mathrm{Te}_{3}$ (the former resembling the crystalline phase; cf. Figure $1 \mathrm{~b}, \mathrm{c})$, and typical four- and five-membered primitive rings in $\mathrm{a}-\mathrm{Sb}_{2} \mathrm{Te}_{3}$. In both amorphous phases, the bonding angles in four-membered rings are close to $90^{\circ}$, whereas higher-membered rings typically include nonoctahedral-like structural fragments.
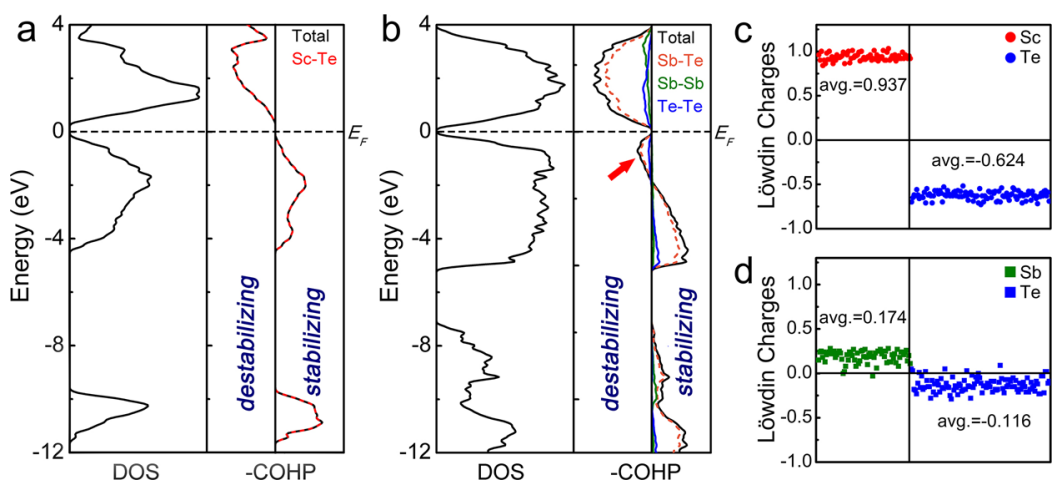

Figure 5. Chemical bonding in $\mathrm{a}-\mathrm{Sc}_{2} \mathrm{Te}_{3}$ and $\mathrm{a}-\mathrm{Sb}_{2} \mathrm{Te}_{3}$. (a,b) DOS and COHP analysis for $\mathrm{a}-\mathrm{Sc}_{2} \mathrm{Te}_{3}$ and a-Sb $\mathrm{Te}_{3}$. Contributions from all atomic contacts up to $3.4 \AA$ were collected for COHP analyses. (c,d) Löwdin charges (in electrons per atom) of a-Sc $\mathrm{Se}_{2} \mathrm{Te}_{3}$ and a-Sb $\mathrm{Te}_{3}$. The $x$-axis is an arbitrary running index, and average values are given for all atomic species.

small peak near $170^{\circ}$ in both compounds (Figure 3b). A major difference between the two structures is that a shoulder and a small peak appear around $70^{\circ}$ and $130^{\circ}$ for Te atoms in a$\mathrm{Sc}_{2} \mathrm{Te}_{3}$, corresponding to the nonoctahedral bonding environments that are observed in the rhombohedral phase of $\mathrm{Sc}_{2} \mathrm{Te}_{3}$. Typical structural motifs of the two phases are shown in Figure 3b. The preponderance of (defective-) octahedral coordination is confirmed by computing the local order parameter, ${ }^{38}$

$$
q=1-\frac{3}{8} \sum_{i>k}\left(\frac{1}{3}+\cos \theta_{i j k}\right)^{2}
$$

where the sum runs over pairs of atoms ( $i$ and $k$ ) bonded to a central atom $(j)$. No tetrahedral motifs $(q>0.8)$ are identified in $\mathrm{a}-\mathrm{Sb}_{2} \mathrm{Te}_{3}$. With regard to $\mathrm{a}-\mathrm{Sc}_{2} \mathrm{Te}_{3}$, all $\mathrm{Sc}$ atoms are octahedrally coordinated, whereas a small fraction of Te atoms $(\sim 5 \%)$ is found in tetrahedral coordination. Overall, the dominant octahedral motifs closely resemble the local structure in the defective rocksalt-like crystals (Figure 1c,d). ${ }^{22,31}$

The analysis of primitive ("shortest-path") rings is typically used to understand the medium-range order of amorphous networks. An ideal rocksalt lattice consists of four-membered rings ( $\mathrm{ABAB}$ squares). In the amorphous phase, more complex rings can emerge. The distribution of primitive rings up to tenfold for the two compounds is shown in Figure 4a. Four- membered rings are the dominant structural fragment in a$\mathrm{Sb}_{2} \mathrm{Te}_{3}$, whereas other even- and odd-membered rings are also observed. By contrast, comparable fractions of four-, six-, eight-, and ten-membered primitive rings are found in a$\mathrm{Sc}_{2} \mathrm{Te}_{3}$, whereas odd-membered rings are completely absent (Figure $4 \mathrm{a}$ ). $\mathrm{ABAB}$ squares with $\mathrm{A}=\mathrm{Sc}$ or $\mathrm{Sb}$ and $\mathrm{B}=\mathrm{Te}$ represent the smallest crystal units in recrystallized SST. In a$\mathrm{Sb}_{2} \mathrm{Te}_{3}, \mathrm{ABAB}$ squares constitute $\sim 80 \%$ of the four-membered primitive rings, whereas rest of them are in $A A A B$ or $A B B B$ forms. But in $\mathrm{a}-\mathrm{Sc}_{2} \mathrm{Te}_{3}$, all four-membered primitive rings are $\mathrm{ABAB}$ squares. These structural differences between the two compounds stem from the absence of homopolar bonds in $\mathrm{Sc}_{2} \mathrm{Te}_{3}$, as to form odd-membered primitive rings, at least one pair of homopolar bonds must be present. Typical ring motifs found in our simulations of $\mathrm{a}-\mathrm{Sc}_{2} \mathrm{Te}_{3}$ and $\mathrm{a}-\mathrm{Sb}_{2} \mathrm{Te}_{3}$ are shown in Figure $4 \mathrm{~b}-\mathrm{e}$. This property explains why, upon alloying $\mathrm{Sc}$ atoms into $\mathrm{Sb}_{2} \mathrm{Te}_{3}$, the number of high-quality crystalline precursors consisting of $\mathrm{ABAB}$ squares increases.

Electronic and Bonding Nature. Next, we carry out the chemical bonding analyses of the two amorphous compounds using the crystal orbital Hamilton population (COHP) method. ${ }^{41-43}$ This method dissects the electronic density of states (DOS) into bonding (stabilizing) interaction and antibonding (destabilizing) interaction, revealing the chemical stability of compounds. Both a- $\mathrm{Sc}_{2} \mathrm{Te}_{3}$ and a-Sb $\mathrm{Te}_{3}$ models 

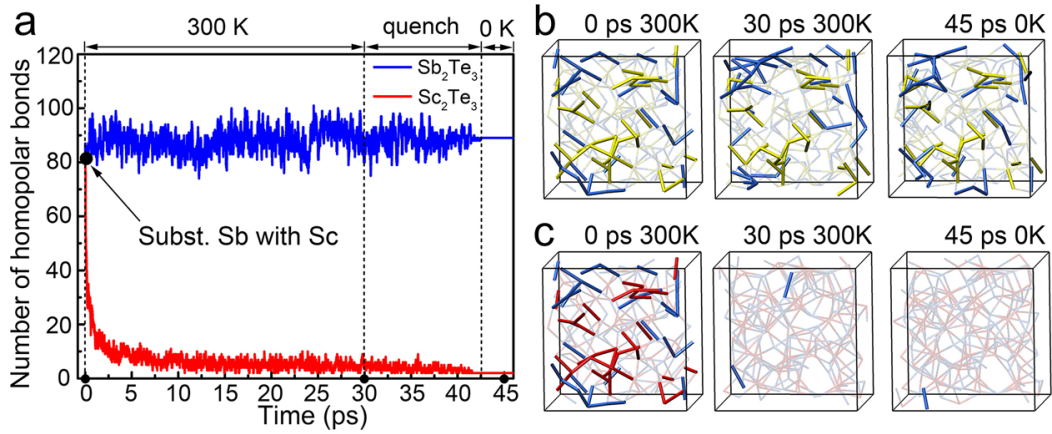

Figure 6. Strong relaxation of a-Sc $\mathrm{Se}_{2}$ in the presence of homopolar bonds. (a) Statistics of homopolar bonds for melt-quenched a-Sb $\mathrm{Te}_{3}$ (blue) and a hypothetical a- $\mathrm{Sc}_{2} \mathrm{Te}_{3}$ structure created by chemical substitution (red). The starting configuration is obtained by substituting all $\mathrm{Sb}$ atoms with $\mathrm{Sc}$ atoms in the $\mathrm{a}-\mathrm{Sb}_{2} \mathrm{Te}_{3}$ model and re-scaling the cell volume to match that of our melt-quenched a-Sc $\mathrm{Te}_{3}$ models. (b,c) Evolution of homopolar bonds (drawn as thick colored sticks) in the simulations. Yellow, red, and blue bonds represent $\mathrm{Sb}-\mathrm{Sb}, \mathrm{Sc}-\mathrm{Sc}$, and $\mathrm{Te}-\mathrm{Te}$ bonds, respectively.
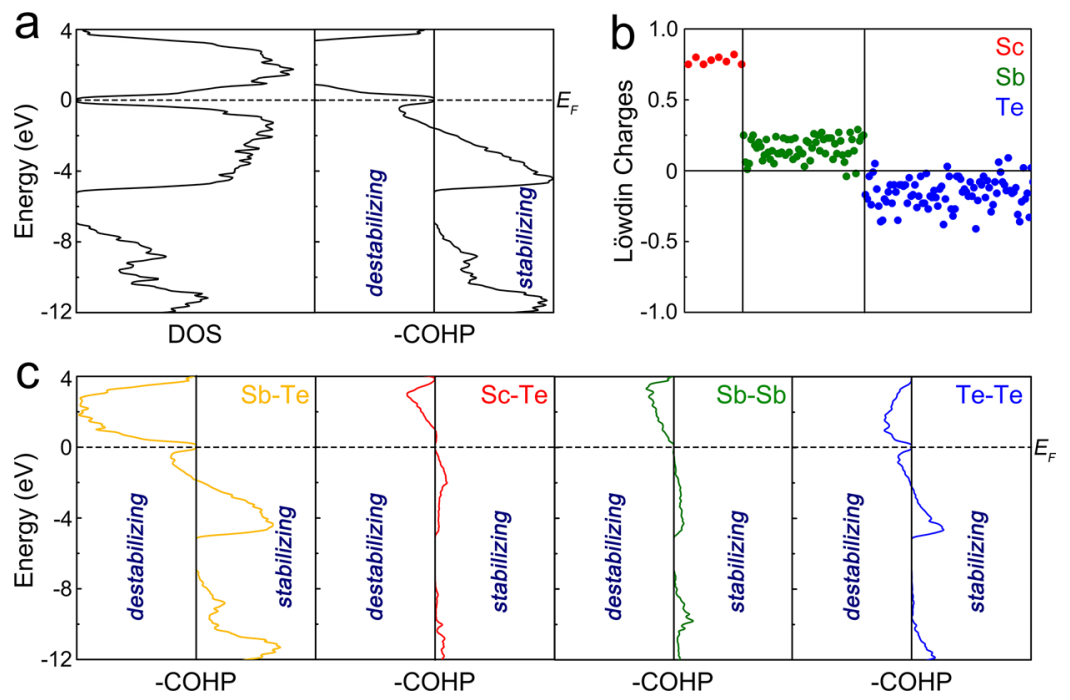

Figure 7. Chemical bonding analysis of a-SST. The model contains $8 \mathrm{Sc}, 64 \mathrm{Sb}$, and $108 \mathrm{Te}$ atoms in a $18.75 \times 18.75 \times 18.75 \AA^{3}$ supercell, corresponding to the theoretical density of the amorphous phase. Both the atomic positions and volume are relaxed at $0 \mathrm{~K}$. (a,b) DOS, COHP, and Löwdin charge analyses for a-SST. (c) COHP plots resolved according to $\mathrm{Sb}-\mathrm{Te}, \mathrm{Sc}-\mathrm{Te}, \mathrm{Sb}-\mathrm{Sb}$, and $\mathrm{Te}-\mathrm{Te}$ bonds, collected over all occurrences of the respective contact in the simulation cell. $\mathrm{Sc}-\mathrm{Sc}$ and $\mathrm{Sc}-\mathrm{Sb}$ bonds are not found in the amorphous models. Contributions from all atomic contacts up to $3.4 \AA ̊$ were collected for COHP analyses.

were quenched down and optimized at $0 \mathrm{~K}$ for the DOS and COHP calculations. DFT calculations (using the PBE functional) show that rhombohedral $\mathrm{Sb}_{2} \mathrm{Te}_{3}$ has a narrow band gap of $0.15 \mathrm{eV},{ }^{44}$ whereas no band gap is found for all three crystalline phases of $\mathrm{Sc}_{2} \mathrm{Te}_{3}$ (Figure S3). With regard to the amorphous phase, finite band gaps are found for both a$\mathrm{Sc}_{2} \mathrm{Te}_{3}(\sim 0.25 \mathrm{eV})$ and $\mathrm{a}-\mathrm{Sb}_{2} \mathrm{Te}_{3}(\sim 0.1 \mathrm{eV})$, see Figure 5a,b. This suggests a metal-to-semiconductor transition for $\mathrm{Sc}_{2} \mathrm{Te}_{3}$ upon amorphization. The closed band gap in rhombohedral $\mathrm{Sc}_{2} \mathrm{Te}_{3}$ is due to defects states brought by the high amount of atomic vacancies, as a finite gap of $\sim 0.2 \mathrm{eV}$ can be obtained if $\mathrm{Sc}_{2} \mathrm{Te}_{3}$ takes the crystal structure of rhombohedral $\mathrm{Sb}_{2} \mathrm{Te}_{3}$, where no vacancies are present (Figure S4). As regards the bonding, both amorphous structures are free from antibonding interaction directly at the Fermi level, indicating good chemical stability of the two phases (Figure 5a,b). For a- $\mathrm{Sc}_{2} \mathrm{Te}_{3}$, all filled bands up to the $E_{\mathrm{F}}$ contribute to stabilization of the system, whereas there exists a finite antibonding interaction region below $E_{\mathrm{F}}$ in $\mathrm{a}-\mathrm{Sb}_{2} \mathrm{Te}_{3}$, indicating a potential source of instability of the system. To make a more direct comparison, we create a hypothetical a-Sb $\mathrm{Te}_{3}$ model to rule out the effects of homopolar bonds: for this, we take an $\mathrm{a}-\mathrm{Sc}_{2} \mathrm{Te}_{3}$ structure and substitute all $\mathrm{Sc}$ atoms with $\mathrm{Sb}$ atoms in exactly the same geometrical configuration, including the atomic position and the lattice parameter, and perform the COHP analysis for the resulting model. In this configuration, all bond contributions are made by $\mathrm{Sb}-\mathrm{Te}$ bonds, and the antibonding interaction below the $E_{\mathrm{F}}$ is still present (Figure S5). Overall, we can conclude that $a-S_{2} \mathrm{Te}_{3}$ is chemically more robust than a$\mathrm{Sb}_{2} \mathrm{Te}_{3}$. These bonding features are in line with those found in the crystalline structures ${ }^{22}$ (Figure S5).

The absence of homopolar bonds leads to better defined crystalline precursors- $\mathrm{ABAB}$ squares and cubes in a-Sc $\mathrm{Te}_{3}$ (Figure $4 \mathrm{~b}$ ). To further understand these structural features, we create a hypothetical a-Sc $\mathrm{Te}_{2}$ model in which all $\mathrm{Sb}$ atoms in an $\mathrm{a}-\mathrm{Sb}_{2} \mathrm{Te}_{3}$ configuration are substituted with $\mathrm{Sc}$ atoms, forming a finite fraction of homopolar $\mathrm{Sc}-\mathrm{Sc}$ and $\mathrm{Te}-\mathrm{Te}$ bonds (Figure 6). The presence of these homopolar bonds leads to a high penalty in total energy of $\sim 600 \mathrm{meV}$ /atom, which drives a strong relaxation of the system even at room temperature (Figure 6c). All of the homopolar bonds vanish quickly at room temperature, forming perfect $A B A B$ squares 
and cubes again. This tendency can be qualitatively understood based on the difference in electronegativity of the constituent elements: this difference is much larger for the pair $\mathrm{Sc} / \mathrm{Te}$ than for $\mathrm{Sb} / \mathrm{Te}$, leading to a stronger charge transfer in $\mathrm{a}-\mathrm{Sc}_{2} \mathrm{Te}_{3}$. More quantitative information about individual atomic charges can be obtained from first-principles calculations: for this purpose, we use the same local-basis projection scheme as for the COHP analysis and now compute Löwdin charges ${ }^{45}$ (Figure 5c). Clearly, in a- $\mathrm{Sc}_{2} \mathrm{Te}_{3}$, atoms are more ionic than in $\mathrm{a}-\mathrm{Sb}_{2} \mathrm{Te}_{3}$, which increases the Coulomb repulsion between atoms with the same type of charge, resulting in a stronger energy penalty. Such ionic character is also present in the crystalline phases of $\mathrm{Sc}_{2} \mathrm{Te}_{3}$ (Figure S3).

Implications for Memory Devices. In typical phasechange memory devices based on GST or SST, the thickness of PCM layers amounts to several tens to a few hundred nanometers, and nucleation plays a leading role for crystallization. ${ }^{6}$ Although not determined precisely, the size of critical nuclei in SST is estimated to be several tens of atoms at $\sim 600 \mathrm{~K}$, corresponding to several connected cubes. ${ }^{22}$ The commercialized GST-based phase-change random access memory (PRAMs) suffer from the stochastic incubation process for nucleation in that the abundant crystalline precursors ( $\mathrm{ABAB}$ squares and cubes) break and reform rapidly with a very short lifetime of $\sim 5$ ps at elevated temperatures, for example $\sim 600 \mathrm{~K}$. By contrast, $\mathrm{Sc}-\mathrm{Te}$ bonds are typically more robust and can stay intact over long periods of time at $600 \mathrm{~K}$. Here, our direct chemical bonding analyses of amorphous SST provide additional theoretical support for this scenario (Figure 7). The overall DOS and COHP curves of aSST look similar to those of $\mathrm{a}-\mathrm{Sb}_{2} \mathrm{Te}_{3}$ in that an antibonding region is found right below the Fermi level (Figure 7a). Resolving the COHP contributions to individual types of neighboring species reveals that this antibonding interaction is mainly contributed by $\mathrm{Sb}-\mathrm{Te}$ and $\mathrm{Te}-\mathrm{Te}$ bonds (Figure $7 \mathrm{c}$ ). However, a stabilizing bonding interaction is observed for ScTe bonds in this energy range (Figure 7c). Moreover, the Löwdin charge analysis shows a more ionic character of Sc atoms in a-SST (Figure 7b), which results in the absence of homopolar Sc-Sc bonds because of the high energy penalty for cation-cation repulsion. The bonding nature of Sc atoms in a-SST generates high-quality and high-strength $\mathrm{Sc}-\mathrm{Te}$ squares and cubes, thereby suppressing the stochasticity of incubation for ultrafast SET operations in SST-based PRAMs.

Our simulations reveal that Sc-centered local motifs in amorphous and all three crystalline states of $\mathrm{Sc}_{2} \mathrm{Te}_{3}$ always exhibit perfect or defective octahedral configuration, whereas a fraction of $\mathrm{Te}$ atoms in both rhombohedral and amorphous states tends to form nonoctahedral bonds, which also result in higher-membered rings in the amorphous structure, deviating from the rocksalt one (Figure 4c). Given the robustness of ScTe bonds, the nonoctahedral bonds and six-membered primitive rings may stay intact over long period of time at $\sim 600 \mathrm{~K}$, hindering crystallization into rocksalt-like structure. Our findings thus indicate that too much addition of Sc should be avoided for high-speed $\mathrm{Sc}-\mathrm{Sb}-\mathrm{Te}$ phase-change memory devices. Given a limited concentration of $\mathrm{Sc}$, such as $4 \%$ atomic percent in SST $\left(10 \% \mathrm{Sc}_{2} \mathrm{Te}_{3}\right.$ and $\left.90 \% \mathrm{Sb}_{2} \mathrm{Te}_{3}\right)$, Te atoms would have a very low probability of forming nonoctahedral $\mathrm{Sc}-\mathrm{Te}-\mathrm{Sc}$ bonds in the Sc-poor environment. Another effect brought by the overconcentration of Sc is an increased viscosity ${ }^{22}$ stemming from the strong, partly-ionic $\mathrm{Sc}-\mathrm{Te}$ chemical bonds. On the one hand, the latter effect stabilizes the amorphous phase at low temperature for longer data retention, but, on the other hand, it reduces the atomic mobility at elevated temperatures, slowing down the crystal growth of SST and impeding the subnanosecond SET operations. The so-derived chemical design principles for high-performance cache-type $\mathrm{Sc}-\mathrm{Sb}-\mathrm{Te} \mathrm{PCM}$ are summarized in Figure 8.

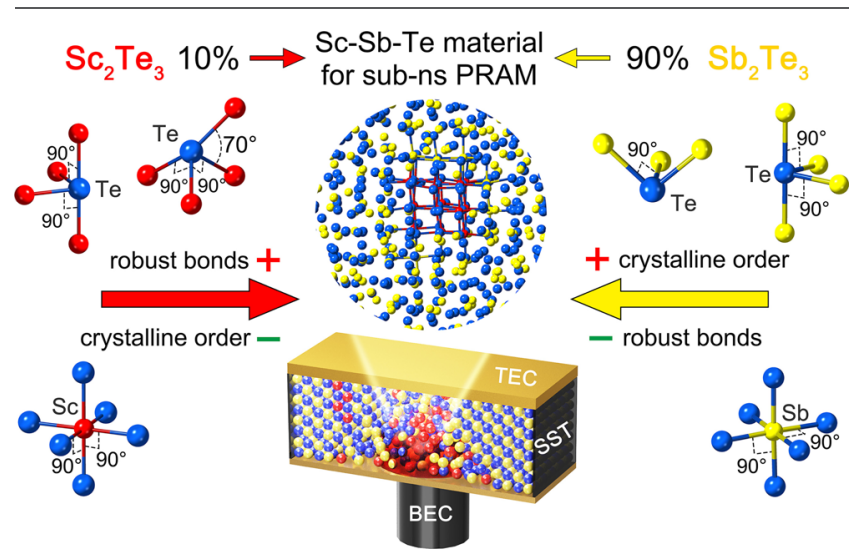

Figure 8. The chemical design principles for cache-type $\mathrm{Sc}-\mathrm{Sb}-\mathrm{Te}$ compounds. When a limited fraction of $\mathrm{Sc}_{2} \mathrm{Te}_{3}(10 \%)$ is alloyed into $\mathrm{Sb}_{2} \mathrm{Te}_{3}(90 \%)$, the SST compound (4\% Sc) can take advantages of both parent compounds to undergo ultrafast nucleation with highquality and robust $\mathrm{Sc}-\mathrm{Te}$ crystalline precursors and subsequent rapid crystal growth of $\mathrm{Sb}-\mathrm{Te}$ components. Thus, subnanosecond SET operations can be achieved in conventional PRAM cells with SST PCM. The top and bottom electrode contact are abbreviated as TEC and BEC.

\section{CONCLUSIONS}

In summary, the structural and chemical bonding properties of amorphous $\mathrm{Sc}_{2} \mathrm{Te}_{3}$ and $\mathrm{Sb}_{2} \mathrm{Te}_{3}$ have been clarified by comprehensive $\mathrm{ab}$ initio simulations. Our structural analyses reveal good geometrical compatibility between the two amorphous phases, whereas $\mathrm{a}-\mathrm{Sc}_{2} \mathrm{Te}_{3}$ has a better defined local geometry. The absence of homopolar bonds in a-Sc $\mathrm{Te}_{3}$ is attributed to strong charge transfer, and to the resulting high energy penalty for homopolar bonds. Although a- $\mathrm{Sc}_{2} \mathrm{Te}_{3}$ exhibits high chemical stability, our simulations also indicate that too large $\mathrm{Sc}$ concentrations in the $\mathrm{Sc}-\mathrm{Sb}-\mathrm{Te}$ alloy need to be avoided, as the increased medium-range order brought by $\mathrm{a}-\mathrm{Sc}_{2} \mathrm{Te}_{3}$ would drive the amorphous structure away from the local order in the targeted crystalline counterparts, increasing structural complexity that impedes rapid nucleation. In contrast, alloying a small amount of $\mathrm{Sc}_{2} \mathrm{Te}_{3}$ into $\mathrm{Sb}_{2} \mathrm{Te}_{3}$, better-defined and chemically more robust $\mathrm{ABAB}$ squares and cubes enable enhanced nucleation in the resulting SST material, thereby enabling subnanosecond memory operations.

\section{METHODS}

The structural relaxation and self-consistent electronic-structure calculations based on DFT were carried out using the Vienna Ab initio simulation package, with a plane wave cutoff of $500 \mathrm{eV}$, the projector augmented-wave pseudopotentials, ${ }^{46}$ and the PBE functional. $^{47}$ All crystalline models are calculated by using the experimental lattice parameters. For rhombohedral and rocksalt $\mathrm{Sc}_{2} \mathrm{Te}_{3}$ and rocksalt $\mathrm{Sb}_{2} \mathrm{Te}_{3}$, three independent models, where the positions of atomic vacancies are generated by a random number generator, are calculated for each crystalline phase. The averaged energy values are taken for the calculation of energy difference. More 
details about the crystalline $\mathrm{Sc}_{2} \mathrm{Te}_{3}$ can be found in Figure S3. The chemical bonding analysis was performed using the COHP method, ${ }^{41-43}$ as implemented in the local orbital basis suite towards electronic-structure reconstruction (LOBSTER). ${ }^{48}$ This bonding analysis tool reads the wave functions from plane-wave DFT calculations, ${ }^{49}$ and projects them onto localized orbitals, thereby making large and complex structural models readily accessible to a chemical interpretation. In the recent years, this approach has been applied to highly disordered crystalline $e^{2,50,51}$ and even amorphous PCMs. ${ }^{52-54}$ More recently, charge analyses have been implemented in LOBSTER, making it possible to assign Löwdin atomic charges based on the same projection scheme and thereby analyzing both the covalent and the electrostatic nature of the system. ${ }^{55}$ DFT based AIMD simulations were carried out using the second-generation CarParrinello scheme, ${ }^{56}$ implemented in the Quickstep code of the CP2K package. ${ }^{57}$ The code uses a mixed scheme of Gaussian-type basis sets and plane waves. The Kohn-Sham orbitals were expanded in basis sets with triple-zeta plus polarization quality. Plane waves with a cutoff of $300 \mathrm{Ry}$ were used to expand the charge density. The Goedecker pseudopotentials ${ }^{58}$ and the PBE functional ${ }^{47}$ were used. The Brillouin zone was sampled at the $\Gamma$ point of the supercell. AIMD simulations were performed in the canonical ensemble $(N V T)$ with a stochastic Langevin thermostat. The time step was 2 fs. During quenching simulations, the size of the supercell was adjusted seven times, and each simulation was an independent NVT run.

\section{ASSOCIATED CONTENT}

\section{S Supporting Information}

The Supporting Information is available free of charge on the ACS Publications website at DOI: 10.1021/acs.chemmater.9b00510.

Peierls-like distortion analysis, schematic descriptions of melt-quench simulations, and chemical bonding and charge analyses for various phases of $\mathrm{Sc}_{2} \mathrm{Te}_{3}$ and $\mathrm{Sb}_{2} \mathrm{Te}_{3}$ (PDF)

\section{AUTHOR INFORMATION}

\section{Corresponding Author}

*E-mail: wzhang0@mail.xjtu.edu.cn.

\section{ORCID $\odot$}

Yuxing Zhou: 0000-0001-8061-3867

Volker L. Deringer: 0000-0001-6873-0278

Wei Zhang: 0000-0002-0720-4781

\section{Author Contributions}

${ }^{\#}$ G.M.Z. and Y.Z. contributed equally.

\section{Notes}

The authors declare no competing financial interest.

\section{ACKNOWLEDGMENTS}

W.Z. thanks the support of National Natural Science Foundation of China (61774123), 111 Project 2.0 (BP2018008), the Science and Technology Department of Jiangsu Province (BK20170414), and the Young Talent Support Plan of Xi'an Jiaotong University. G.M.Z. acknowledges scholarship support from Chinese Scholarship Council. V.L.D. acknowledges a Leverhulme Early Career Fellowship and support from the Isaac Newton Trust. R.M. acknowledges funding from Deutsche Forschungsgemeinschaft within SFB 917 "Nanoswitches". F.R. gratefully thanks the National Natural Science Foundation of China (61622408), the Major Provincial Basic Research Program of Guangdong (2017KZDXM070), and the Science and Technology Foundation of Shenzhen (JCYJ20180507182248605, JCYJ20170302150053136). The authors also acknowledge the support by the HPC platform of Xi'an Jiaotong University and the International Joint Laboratory for Micro/Nano Manufacturing and Measurement Technologies of Xi'an Jiaotong University.

\section{REFERENCES}

(1) Wong, H.-S. P.; Salahuddin, S. Memory leads the way to better computing. Nat. Nanotechnol. 2015, 10, 191-194.

(2) Wuttig, M.; Yamada, N. Phase-change materials for rewriteable data storage. Nat. Mater. 2007, 6, 824-832.

(3) Wong, H.-S. P.; Raoux, S.; Kim, S.; Liang, J.; Reifenberg, J. P.; Rajendran, B.; Asheghi, M.; Goodson, K. E. Phase Change Memory. Proc. IEEE 2010, 98, 2201.

(4) Zhang, W.; Ma, E. Single-element glass to record data. Nat. Mater. 2018, 17, 654-655.

(5) Salinga, M.; Kersting, B.; Ronneberger, I.; Jonnalagadda, V. P.; Vu, X. T.; Le Gallo, M.; Giannopoulos, I.; Cojocaru-Mirédin, O.; Mazzarello, R.; Sebastian, A. Monatomic phase change memory. Nat. Mater. 2018, 17, 681-685.

(6) Zhang, W.; Mazzarello, R.; Wuttig, M.; Ma, E. Designing crystallization in phase-change materials for universal memory and neuro-inspired computing. Nat. Rev. Mater. 2019, 4, 150-168.

(7) Kuzum, D.; Jeyasingh, R. G. D.; Lee, B.; Wong, H.-S. P. Nanoelectronic programmable synapses based on phase change materials for brain-inspired computing. Nano Lett. 2012, 12, 21792186.

(8) Ambrogio, S.; Narayanan, P.; Tsai, H.; Shelby, R. M.; Boybat, I.; di Nolfo, C.; Sidler, S.; Giordano, M.; Bodini, M.; Farinha, N. C. P.; Killeen, B.; Cheng, C.; Jaoudi, Y.; Burr, G. W. Equivalent-accuracy accelerated neural-network training using analogue memory. Nature 2018, 558, 60-67.

(9) Tuma, T.; Pantazi, A.; Le Gallo, M.; Sebastian, A.; Eleftheriou, E. Stochastic phase-change neurons. Nat. Nanotechnol. 2016, 11, 693699.

(10) Fong, S. W.; Neumann, C. M.; Wong, H.-S. P. Phase-Change Memory-Towards a Storage-Class Memory. IEEE Trans. Electron Devices 2017, 64, 4374-4385.

(11) Hruska, J. Intel Announces New Optane DC Persistent Memory. https://www.extremetech.com/extreme/270270-intelannounces-new-optane-dc-persistent-memory (accessed May 31, 2018).

(12) Yamada, N.; Ohno, E.; Nishiuchi, K.; Akahira, N.; Takao, M. Rapid-phase transitions of GeTe-Sb2Te3pseudobinary amorphous thin films for an optical disk memory. J. Appl. Phys. 1991, 69, 28492856.

(13) Kolobov, A. V.; Fons, P.; Frenkel, A. I.; Ankudinov, A. L.; Tominaga, J.; Uruga, T. Understanding the phase-change mechanism of rewritable optical media. Nat. Mater. 2004, 3, 703-708.

(14) Sun, Z.; Zhou, J.; Ahuja, R. Structure of Phase Change Materials for Data Storage. Phys. Rev. Lett. 2006, 96, 055507.

(15) Kooi, B. J.; Groot, W. M. G.; De Hosson, J. T. M. In situtransmission electron microscopy study of the crystallization of Ge2Sb2Te5. J. Appl. Phys. 2004, 95, 924.

(16) Wang, J.-J.; Wang, J.; Du, H.; Lu, L.; Schmitz, P. C.; Reindl, J.; Mio, A. M.; Jia, C.-L.; Ma, E.; Mazzarello, R.; Wuttig, M.; Zhang, W. Genesis and Effects of Swapping Bilayers in Hexagonal GeSb2Te4. Chem. Mater. 2018, 30, 4770-4777.

(17) Loke, D.; Lee, T. H.; Wang, W. J.; Shi, L. P.; Zhao, R.; Yeo, Y. C.; Chong, T. C.; Elliott, S. R. Breaking the speed limits of phasechange memory. Science 2012, 336, 1566-1569.

(18) Orava, J.; Greer, A. L.; Gholipour, B.; Hewak, D. W.; Smith, C. E. Characterization of supercooled liquid $\mathrm{Ge}_{2} \mathrm{Sb}_{2} \mathrm{Te}_{5}$ and its crystallization by ultrafast-heating calorimetry. Nat. Mater. 2012, 11, $279-283$.

(19) Zalden, P.; von Hoegen, A.; Landreman, P.; Wuttig, M.; Lindenberg, A. M. How Supercooled Liquid Phase-Change Materials Crystallize: Snapshots after Femtosecond Optical Excitation. Chem. Mater. 2015, 27, 5641-5646. 
(20) Behrens, M.; Lotnyk, A.; Gerlach, J. W.; Hilmi, I.; Abel, T.; Lorenz, P.; Rauschenbach, B. Ultrafast interfacial transformation from 2D- to 3D-bonded structures in layered Ge-Sb-Te thin films and heterostructures. Nanoscale 2018, 10, 22946-22953.

(21) Loke, D. K.; Skelton, J. M.; Lee, T. H.; Zhao, R.; Chong, T.-C.; Elliott, S. R. Ultrafast Nanoscale Phase-Change Memory Enabled By Single-Pulse Conditioning. ACS Appl. Mater. Interfaces 2018, 10, 41855-41860.

(22) Rao, F.; Ding, K.; Zhou, Y.; Zheng, Y.; Xia, M.; Lv, S.; Song, Z.; Feng, S.; Ronneberger, I.; Mazzarello, R.; Zhang, W.; Ma, E. Reducing the stochasticity of crystal nucleation to enable subnanosecond memory writing. Science 2017, 358, 1423-1427.

(23) Wuttig, M. Towards a universal memory? Nat. Mater. 2005, 4, 265-266.

(24) Kohara, S.; Kato, K.; Kimura, S.; Tanaka, H.; Usuki, T.; Suzuya, K.; Tanaka, H.; Moritomo, Y.; Matsunaga, T.; Yamada, N.; Tanaka, Y.; Suematsu, H.; Takata, M. Structural basis for the fast phase change of Ge2Sb2Te5: Ring statistics analogy between the crystal and amorphous states. Appl. Phys. Lett. 2006, 89, 201910.

(25) Akola, J.; Jones, R. Structural phase transitions on the nanoscale: The crucial pattern in the phase-change materials Ge2Sb2Te5 and GeTe. Phys. Rev. B: Condens. Matter Mater. Phys. 2007, 76, 235201.

(26) Hegedüs, J.; Elliott, S. R. Microscopic origin of the fast crystallization ability of $\mathrm{Ge}-\mathrm{Sb}$-Te phase-change memory materials. Nat. Mater. 2008, 7, 399-405.

(27) Ronneberger, I.; Zhang, W.; Eshet, H.; Mazzarello, R. Crystallization properties of the $\mathrm{Ge}_{2} \mathrm{Sb}_{2} \mathrm{Te}_{5}$ phase-change compound from advanced simulations. Adv. Funct. Mater. 2015, 25, 6407-6413.

(28) Ronneberger, I.; Zhang, W.; Mazzarello, R. Crystal growth of $\mathrm{Ge}_{2} \mathrm{Sb}_{2} \mathrm{Te}_{5}$ at high temperatures. MRS Commun. 2018, 8, 1018-1023.

(29) Xu, M.; Cheng, Y.; Sheng, H.; Ma, E. Nature of Atomic Bonding and Atomic Structure in the Phase-Change $\mathrm{Ge}_{2} \mathrm{Sb}_{2} \mathrm{Te}_{5}$ Glass. Phys. Rev. Lett. 2009, 103, 195502.

(30) Belotskii, D. P.; Babyuk, P. F. The $\mathrm{In}_{2} \mathrm{Te}_{3}-\mathrm{Sb}_{2} \mathrm{Te}_{3}$ system. Inorg. Chem. 1970, 6, 505-507.

(31) Zheng, Y.; Xia, M.; Cheng, Y.; Rao, F.; Ding, K.; Liu, W.; Jia, Y.; Song, Z.; Feng, S. Direct observation of metastable face-centered cubic $\mathrm{Sb}_{2} \mathrm{Te}_{3}$ crystal. Nano Res. 2016, 9, 3453-3462.

(32) Yamada, N.; Matsunaga, T. Structure of laser-crystallized $\mathrm{Ge}_{2} \mathrm{Sb}_{2}+\mathrm{xTe} \mathrm{e}_{5}$ sputtered thin films for use in optical memory. J. Appl. Phys. 2000, 88, 7020-7028.

(33) Zhang, B.; Zhang, W.; Shen, Z.; Chen, Y.; Li, J.; Zhang, S.; Zhang, Z.; Wuttig, M.; Mazzarello, R.; Ma, E.; Han, X. Elementresolved atomic structure imaging of rocksalt $\mathrm{Ge}_{2} \mathrm{Sb}_{2} \mathrm{Te}_{5}$ phasechange material. Appl. Phys. Lett. 2016, 108, 191902.

(34) Lotnyk, A.; Bernütz, S.; Sun, X.; Ross, U.; Ehrhardt, M.; Rauschenbach, B. Real-space imaging of atomic arrangement and vacancy layers ordering in laser crystallised $\mathrm{Ge}_{2} \mathrm{Sb}_{2} \mathrm{Te}_{5}$ phase change thin films. Acta Mater. 2016, 105, 1-8.

(35) Men'kov, A. A.; Komissarova, L. N.; Simanov, Y. P.; Spicyn, V. I. Scandium Chalcogenides. Dokl. Akad. Nauk SSSR 1961, 141, 364367.

(36) Assoud, A.; Kleinke, H. The Sesquitelluride $\mathrm{Sc}_{2} \mathrm{Te}_{3}$. Acta Crystallogr., Sect. E: Struct. Rep. Online 2006, 62, i17-i18.

(37) White, J. G.; Dismukes, J. P. The Crystal Structure of Scandium Sesquitelluride. Inorg. Chem. 1965, 4, 1760-1763.

(38) Caravati, S.; Bernasconi, M.; Parrinello, M. First-principles study of liquid and amorphous $\mathrm{Sb}_{2} \mathrm{Te}_{3}$. Phys. Rev. B: Condens. Matter Mater. Phys. 2010, 81, 014201.

(39) Zhang, W.; Ronneberger, I.; Li, Y.; Mazzarello, R. Ab initio investigation of amorphous $\mathrm{Sb}_{2}$ Te. Chem. Mon. 2014, 145, 97-101.

(40) Guo, Y. R.; Dong, F.; Qiao, C.; Wang, J. J.; Wang, S. Y.; Xu, M.; Zheng, Y. X.; Zhang, R. J.; Chen, L. Y.; Wang, C. Z.; Ho, K. M. Structural signature and transition dynamics of $\mathrm{Sb}_{2} \mathrm{Te}_{3}$ melt upon fast cooling. Phys. Chem. Chem. Phys. 2018, 20, 11768-11775.

(41) Dronskowski, R.; Bloechl, P. E. Crystal orbital Hamilton populations (COHP): energy-resolved visualization of chemical bonding in solids based on density-functional calculations. J. Phys. Chem. 1993, 97, 8617-8624.

(42) Maintz, S.; Deringer, V. L.; Tchougréeff, A. L.; Dronskowski, R. Analytic projection from plane-wave and PAW wavefunctions and application to chemical-bonding analysis in solids. J. Comput. Chem. 2013, 34, 2557-2567.

(43) Deringer, V. L.; Tchougréeff, A. L.; Dronskowski, R. Crystal orbital Hamilton population (COHP) analysis as projected from plane-wave basis sets. J. Phys. Chem. A 2011, 115, 5461-5466.

(44) Sosso, G. C.; Caravati, S.; Bernasconi, M. Vibrational properties of crystalline $\mathrm{Sb}_{2} \mathrm{Te}_{3}$ from first principles. J. Phys.: Condens. Matter 2009, 21, 095410.

(45) Löwdin, P. O. On the Non-Orthogonality Problem Connected with the Use of Atomic Wave Functions in the Theory of Molecules and Crystals. J. Chem. Phys. 1950, 18, 365-375.

(46) Kresse, G.; Joubert, D. From ultrasoft pseudopotentials to the projector augmented-wave method. Phys. Rev. B: Condens. Matter Mater. Phys. 1999, 59, 1758.

(47) Perdew, J. P.; Burke, K.; Ernzerhof, M. Generalized Gradient Approximation Made Simple. Phys. Rev. Lett. 1996, 77, 3865-3868.

(48) Maintz, S.; Deringer, V. L.; Tchougréeff, A. L.; Dronskowski, R. LOBSTER: A tool to extract chemical bonding from plane-wave based DFT. J. Comput. Chem. 2016, 37, 1030-1035.

(49) Kresse, G.; Hafner, J. Ab initiomolecular dynamics for liquid metals. Phys. Rev. B: Condens. Matter Mater. Phys. 1993, 47, 558-561.

(50) Wuttig, M.; Lüsebrink, D.; Wamwangi, D.; Wełnic, W.; Gillessen, M.; Dronskowski, R. The role of vacancies and local distortions in the design of new phase-change materials. Nat. Mater. 2007, 6, 122-128.

(51) Deringer, V. L.; Zhang, W.; Rausch, P.; Mazzarello, R.; Dronskowski, R.; Wuttig, M. A chemical link between Ge-Sb-Te and In-Sb-Te phase-change materials. J. Mater. Chem. C 2015, 3, 95199523.

(52) Deringer, V. L.; Zhang, W.; Lumeij, M.; Maintz, S.; Wuttig, M.; Mazzarello, R.; Dronskowski, R. Bonding nature of local structural motifs in amorphous GeTe. Angew. Chem., Int. Ed. 2014, 53, 1081710820.

(53) Deringer, V. L.; Dronskowski, R.; Wuttig, M. Microscopic Complexity in Phase-Change Materials and its Role for Applications. Adv. Funct. Mater. 2015, 25, 6343-6359.

(54) Konze, P. M.; Dronskowski, R.; Deringer, V. L. Exploring Chemical Bonding in Phase-Change Materials with Orbital-Based Indicators. Phys. Status Solidi RRL 2019, 13, 1800579.

(55) Li, W.-L.; Ertural, C.; Bogdanovski, D.; Li, J.; Dronskowski, R. Chemical Bonding of Crystalline LnB6 ( $\mathrm{Ln}=\mathrm{La}-\mathrm{Lu}$ ) and Its Relationship with Ln2B8 Gas-Phase Complexes. Inorg. Chem. 2018, 57, 12999-13008.

(56) Kühne, T.; Krack, M.; Mohamed, F.; Parrinello, M. Efficient and Accurate Car-Parrinello-like Approach to Born-Oppenheimer Molecular Dynamics. Phys. Rev. Lett. 2007, 98, 066401.

(57) Hutter, J.; Iannuzzi, M.; Schiffmann, F.; VandeVondele, J. cp2k: atomistic simulations of condensed matter systems. Wiley Interdiscip. Rev.: Comput. Mol. Sci. 2014, 4, 15-25.

(58) Goedecker, S.; Teter, M.; Hutter, J. Separable dual-space Gaussian pseudopotentials. Phys. Rev. B: Condens. Matter Mater. Phys. 1996, 54, 1703. 